\title{
An almost sure central limit theorem of products of partial sums for $\rho^{-}$-mixing sequences
}

\author{
Xili Tan ${ }^{*}$, Ying Zhang ${ }^{1}$ and Yong Zhang ${ }^{2}$
}

\footnotetext{
* Correspondence: tanx10832@sina. com

'Department of Mathematics, Beihua University, Jilin 132013, P.R. China

Full list of author information is available at the end of the article
}

\section{Abstract}

Let $\left\{X_{n}, n \geq 1\right\}$ be a strictly stationary $\rho$-mixing sequence of positive random variables with $E X_{1}=\mu>0$ and $\operatorname{Var}\left(X_{1}\right)=\sigma^{2}<\infty$. Denote $S_{n}=\sum_{i=1}^{n} X_{i}$ and $\gamma=\frac{\sigma}{\mu}$ the coefficient of variation. Under suitable conditions, by the central limit theorem of weighted sums and the moment inequality we show that

$$
\forall x=\lim _{n \rightarrow \infty} \frac{1}{\log n} \sum_{k=1}^{n} \frac{1}{k} I\left\{\left(\prod_{i=1}^{k} \frac{S_{i}}{i \mu}\right)^{\frac{1}{\gamma \sigma k}}\right\}=F(x) \text { a.s. }
$$

where $\sigma_{k}^{2}=\operatorname{Var}\left(S_{k, k}\right), \quad S_{k, k}=\sum_{i=1}^{k} b_{i, k} Y_{i}, \quad b_{i, k}=\sum_{j=i}^{k} \frac{1}{j}, i \leq k$ with

$b_{i, k}=0, i>k, Y_{i}=\frac{X_{i-\mu}}{\sigma}, F(x)$ is the distribution function of the random variable $e^{\sqrt{2} \mathcal{N}}$, and $\mathcal{N}$ is a standard normal random variable.

MR(2000) Subject Classification: 60F15.

Keywords: almost sure central limit theorem, $\rho$ ? $?$ ?-mixing, products of partial sums

\section{Introduction and main results}

For a random variable $X$, define $\|X\|_{p}=\left(E|X|^{p}\right)^{1 / p}$. For two nonempty disjoint sets $S, T$ $\subset N$, we define $\operatorname{dist}(S, T)$ to be $\min \{|j-k| ; j \in S, k \in T\}$. Let $\sigma(S)$ be the $\sigma$-field generated by $\left\{X_{k}, k \in S\right\}$, and define $\sigma(T)$ similarly. Let $\mathscr{C}$ be a class of functions which are coordinatewise increasing. For any real number $x, x^{+}$, and $x^{-}$denote its positive and negative part, respectively, (except for some special definitions, for examples, $\rho^{-}(s), \rho^{-}$ $(S, T)$, etc.). For random variables $X, Y$, define

$$
\rho^{-}(X, Y)=0 \vee \sup \frac{\operatorname{Cov}(f(X), g(Y))}{(\operatorname{Varf}(X))^{\frac{1}{2}}(\operatorname{Varg}(Y))^{\frac{1}{2}}},
$$

where the sup is taken over all $f, g \in \mathscr{C}$ such that $E(f(X))^{2}<\infty$ and $E(g(Y))^{2}<\infty$. A sequence $\left\{X_{n}, n \geq 1\right\}$ is called negatively associated (NA) if for every pair of disjoint subsets $S, T$ of $N$,

$$
\operatorname{Cov}\left\{f\left(X_{i}, i \in S\right), g\left(X_{j}, j \in T\right)\right\} \leq 0,
$$

\section{空

(c) 2012 Tan et al.; licensee Springer. This is an open access article distributed under the terms of the Creative Commons Attribution License (http://creativecommons.org/licenses/by/2.0), which permits unrestricted use, distribution, and reproduction in any medium, provided the original work is properly cited. 
whenever $f, g \in \mathscr{C}$.

A sequence $\left\{X_{n}, n \geq 1\right\}$ is called $\rho^{*}$-mixing if

$$
\rho *(s)=\sup \{\rho(S, T) ; S, T \subset N, \operatorname{dist}(S, T) \geq s\} \rightarrow 0 \text { as } s \rightarrow \infty,
$$

where

$$
\rho(S, T)=\sup \left\{\left|E(f-E f)(g-E g) /\left(\|f-E f\|_{2} \cdot\|g-E g\|_{2}\right)\right| ; f \in L_{2}(\sigma(S)), g \in L_{2}(\sigma(T))\right\} .
$$

A sequence $\left\{X_{n}, n \geq 1\right\}$ is called $\rho^{-}$-mixing, if

$$
\rho^{-}(s)=\sup \left\{\rho^{-}(S, T) ; S, T \subset N, \operatorname{dist}(S, T) \geq s\right\} \rightarrow 0 \text { as } s \rightarrow \infty .
$$

where,

$$
\rho^{-}(S, T)=0 \vee \sup \left\{\frac{\operatorname{Cov}\left\{f\left(X_{i}, i \in S\right), g\left(X_{i}, j \in T\right)\right\}}{\left.\sqrt{\operatorname{Var}\left\{f\left(X_{i}, i \in S\right)\right\} \operatorname{Var}\left\{g\left(X_{i}, j \in T\right)\right.}\right\}} ; f, g \in C\right\} .
$$

The concept of $\rho^{-}$-mixing random variables was proposed in 1999 (see [1]). Obviously, $\rho^{-}$-mixing random variables include NA and $\rho^{*}$-mixing random variables, which have a lot of applications, their limit properties have aroused wide interest recently, and a lot of results have been obtained, such as the weak convergence theorems, the central limit theorems of random fields, Rosenthal-type moment inequality, see [1-4]. Zhou [5] studied the almost sure central limit theorem of $\rho^{-}$-mixing sequences by the conditions provided by Shao: on the conditions of central limit theorem, and if $\varepsilon_{0}>0, \operatorname{Var}\left(\sum_{i=1}^{n} \frac{1}{i} f\left(\frac{S_{i}}{\sigma_{i}}\right)\right)=O\left(\log ^{2-\varepsilon_{0}} n\right)$, where $f$ is Lipschitz function. In this article, we study the almost sure central limit theorem of products of partial sums for $\rho^{-}$-mixing sequence by the central limit theorem of weighted sums and moment inequality.

Here and in the sequel, let $b_{k, n}=\sum_{i=k}^{n} \frac{1}{i}, k \leq n$ with $b_{k, n}=0, k>n$. Suppose $\left\{X_{n}, n \geq 1\right\}$ be a strictly stationary $\rho^{-}$-mixing sequence of positive random variables with $E X_{1}=\mu>$ 0 and $\operatorname{Var}\left(X_{1}\right)=\sigma^{2}<\infty$. Let $\tilde{S}_{n}=\sum_{k=1}^{n} Y_{k}$ and $S_{n, n}=\sum_{k=1}^{n} b_{k, n} Y_{k}$, where $Y_{k}=\frac{X_{k}-\mu}{\sigma}, k \geq 1$. Let $\sigma_{n}^{2}=\operatorname{Var}\left(S_{n, n}\right)$, and $C$ denotes a positive constant, which may take different values whenever it appears in different expressions. The following are our main results.

Theorem 1.1 Let $\left\{X_{n}, n \geq 1\right\}$ be a defined as above with $0<E\left|X_{1}\right|^{r}<\infty$ for a certain $r>2$, denote $S_{n}=\sum_{i=1}^{n} X_{i}$ and $\gamma=\frac{\sigma}{\mu}$ the coefficient of variation. Assume that

$$
\left(a_{1}\right) \sigma_{1}^{2}=E X_{1}^{2}+2 \sum_{n=2}^{\infty} \operatorname{Cov}\left(X_{1}, X_{n}\right)>0,
$$

$\left(a_{2}\right) \sum_{n=2}^{\infty}\left|\operatorname{Cov}\left(X_{1}, X_{n}\right)\right|<\infty$,

$\left(a_{3}\right) \rho^{-}(n)=O\left(\log ^{-\delta} n\right), \exists \delta>1$,

$\left(a_{4}\right) \inf _{n \in N} \frac{\sigma_{n}^{2}}{n}>0$.

Then

$$
\forall x \quad \lim _{n \rightarrow \infty} \frac{1}{\log n} \sum_{k=1}^{n} \frac{1}{k} I\left\{\frac{S_{k, k}}{\sigma_{k}} \leq x\right\}=\Phi(x) \text { a.s. }
$$


Here and in the sequel, $I\{\cdot\}$ denotes indicator function and $\Phi(\cdot)$ is the distribution function of standard normal random variable $\mathcal{N}$.

Theorem 1.2 Under the conditions of Theorem 1.1, then

$$
\forall x=\lim _{n \rightarrow \infty} \frac{1}{\log n} \sum_{k=1}^{n} \frac{1}{k} I\left\{\left(\prod_{i=1}^{k} \frac{S_{i}}{i \mu}\right)^{\frac{1}{\gamma \sigma k}} \leq x\right\}=F(x) \text { a.s. }
$$

Here and in the sequel, $F(\cdot)$ is the distribution function of the random variable $e^{\sqrt{2} \mathcal{N}}$.

\section{Some lemmas}

To prove our main results, we need the following lemmas.

Lemma 2.1 [3] Let $\left\{X_{n}, n \geq 1\right\}$ be a weakly stationary $\rho^{-}$-mixing sequence with $E X_{n}=0, \quad 0<E X_{1}^{2}<\infty$, and

(i) $\sigma_{1}^{2}=E X_{1}^{2}+2 \sum_{n=2}^{\infty} \operatorname{Cov}\left(X_{1}, X_{n}\right)>0$,

(ii) $\sum_{n=2}^{\infty}\left|\operatorname{Cov}\left(X_{1}, X_{n}\right)\right|<\infty$,

then

$$
\frac{E S_{n}^{2}}{n} \rightarrow \sigma_{1}^{2}, \quad \frac{S_{n}}{\sigma_{1} \sqrt{n}} \stackrel{d}{\rightarrow} \mathcal{N}(0,1) \text { as } n \rightarrow \infty
$$

Lemma 2.2 [4] For a positive real number $q \geq 2$, if $\left\{X_{n}, n \geq 1\right\}$ is a sequence of $\rho^{\text {- }}$ mixing random variables with $E X_{i}=0, E\left|X_{i}\right|^{q}<\infty$ for every $i \geq 1$, then for all $n \geq 1$, there is a positive constant $C=C\left(q, \rho^{-}(\cdot)\right)$ such that

$$
E\left(\max _{1 \leq j \leq n}\left|S_{j}\right|^{q}\right) \leq C\left\{\sum_{i=1}^{n} E\left|X_{i}\right|^{q}+\left(\sum_{i=1}^{n} E X_{i}^{2}\right)^{\frac{q}{2}}\right\} .
$$

Lemma $2.3[6] \sum_{i=1}^{n} b_{i, n}^{2}=2 n-b_{1, n}$.

Lemma 2.4 [[3], Theorem 3.2] Let $\left\{X_{n i}, 1 \leq i \leq n, n \geq 1\right\}$ be an array of centered random variables with $E X_{n i}^{2}<\infty$ for each $i=1,2, \ldots, n$. Assume that they are $\rho^{-}$-mixing. Let $\left\{a_{n i}, 1 \leq i \leq n, n \geq 1\right\}$ be an array of real numbers with $a_{n i}= \pm 1$ for $i=1,2, \ldots, n$. Denote $\sigma_{n}^{2}=\operatorname{Var}\left(\sum_{i=1}^{n} a_{n i} X_{n i}\right)$ and suppose that

$$
\sup _{n} \frac{1}{\sigma_{n}^{2}} \sum_{i=1}^{n} E X_{n i}^{2}<\infty
$$

and

$$
\limsup _{n \rightarrow \infty} \frac{1}{\sigma_{n}^{2}} \sum_{\substack{i, j:|i-j| \geq A \\ \text { i } \leq i, j \leq n}} \operatorname{Cov}\left(X_{n i}, X_{n j}\right)^{-} \rightarrow 0 \text { as } A \rightarrow \infty,
$$


and the following Lindeberg condition is satisfied:

$$
\frac{1}{\sigma_{n}^{2}} \sum_{i=1}^{n} E X_{n i}^{2}\left\{\left|X_{n i}\right| \geq \varepsilon \sigma_{n}\right\} \rightarrow 0 \text { as } n \rightarrow \infty
$$

for every $\varepsilon>0$. Then

$$
\frac{1}{\sigma_{n}} \sum_{i=1}^{n} a_{n i} X_{n i} \stackrel{d}{\rightarrow} \mathcal{N}(0,1) \text { as } n \rightarrow \infty .
$$

Lemma 2.5 Let $\left\{X_{n}, n \geq 1\right\}$ be a strictly stationary sequence of $\rho^{-}$-mixing random variables with $E X_{n}=0$ and $\sum_{n=2}^{\infty}\left|\operatorname{Cov}\left(X_{1}, X_{n}\right)\right|<\infty,\left\{a_{n i}, 1 \leq i \leq n, n \geq 1\right\}$ be an array of real numbers such that $\sup _{n} \sum_{i=1}^{n} a_{n i}^{2}<\infty$ and $\max _{1 \leq i \leq n}\left|a_{n i}\right| \rightarrow 0$ as $n \rightarrow \infty$. If $\operatorname{Var}\left(\sum_{i=1}^{n} a_{n i} X_{i}\right)=1$ and $\left\{X_{n}^{2}\right\}$ is an uniformly integrable family, then

$$
\sum_{i=1}^{n} a_{n i} X_{i} \stackrel{d}{\rightarrow} \mathcal{N}(0,1) \text { as } n \rightarrow \infty .
$$

Proof Notice that

$$
\sum_{i=1}^{n} a_{n i} X_{i}=\sum_{i=1}^{n} \frac{a_{n i}}{\left|a_{n i}\right|}\left|a_{n i}\right| X_{i}=: \sum_{i=1}^{n} b_{n i} Y_{n i}
$$

where $b_{n i}=\frac{a_{n i}}{\left|a_{n i}\right|}$ and $Y_{n i}=\left|a_{n i}\right| X_{i}$. Then $\left\{Y_{n i}, 1 \leq i \leq n, n \geq 1\right\}$ is an array of $\rho^{-}$-mixing centered random variables with $E Y_{n i}^{2}=a_{n i}^{2} E X_{i}^{2}<\infty$ and $b_{n i}= \pm 1$ for $i=1,2, \ldots, n$ and $\sigma_{n}^{2}=\operatorname{Var}\left(\sum_{i=1}^{n} b_{n i} Y_{n i}\right)=1$. Note that $\left\{X_{n}^{2}\right\}$ is an uniformly integrable family, we have

$$
\sup _{n} \frac{1}{\sigma_{n}^{2}} \sum_{i=1}^{n} E Y_{n i}^{2}=\sup _{n} \sum_{i=1}^{n} a_{n i}^{2} E X_{i}^{2} \leq \sup _{n} \sum_{i=1}^{n} a_{n i}^{2} \cdot \sup _{i} E X_{i}^{2}<\infty \text {, }
$$


and

$$
\begin{aligned}
& \limsup _{n \rightarrow \infty} \frac{1}{\sigma_{n}^{2}} \sum_{\substack{i, j:|i-j| \geq A \\
1 \leq i, j \leq n}} \operatorname{Cov}\left(Y_{n i}, Y_{n j}\right)^{-} \\
= & \limsup _{n \rightarrow \infty} \sum_{\substack{i, j:|i-j| \geq A \\
1 \leq i, j \leq n}} \operatorname{Cov}\left(\left|a_{n i}\right| X_{i},\left|a_{n j}\right| X_{j}\right)^{-} \\
\leq & \limsup _{n \rightarrow \infty} \sum_{\substack{i, j:|i-j| \geq A \\
1 \leq i, j \leq n}}\left|a_{n i}\right| \cdot\left|a_{n j}\right| \cdot\left|\operatorname{Cov}\left(X_{i}, X_{j}\right)\right| \\
\leq & \left.C \limsup _{n \rightarrow \infty} \sum_{\substack{i, j:|i-j| \geq A \\
1 \leq i, j \leq n}}\left|a_{n i}\right|^{2} \cdot\left|\operatorname{Cov}\left(X_{i}, X_{j}\right)\right|+\sum_{\substack{i, j:|i-j| \geq A \\
1 \leq i, j \leq n}}\left|a_{n i}\right|^{2} \cdot\left|\operatorname{Cov}\left(X_{i}, X_{j}\right)\right|\right) \\
\leq & C \sup _{n} \sum_{i=1}^{n}\left|a_{n i}\right|^{2} \cdot \sum_{i>A}\left|\operatorname{Cov}\left(X_{1}, X_{i}\right)\right| \\
\rightarrow & 0 \operatorname{ass}_{A \rightarrow \infty} A,
\end{aligned}
$$

and $\forall \varepsilon>0$, we get

$$
\begin{aligned}
& \frac{1}{\sigma_{n}^{2}} \sum_{i=1}^{n} E Y_{n i}^{2} I\left\{\left|Y_{n i}\right| \geq \varepsilon \sigma_{n}\right\} \\
= & \sum_{i=1}^{n} a_{n i}^{2} E X_{i}^{2} I\left\{\left|a_{n i}\right| \cdot\left|X_{i}\right| \geq \varepsilon\right\} \\
\leq & \sup _{n} \sum_{i=1}^{n} a_{n i}^{2} \cdot E X_{1}^{2} I\left\{\left|a_{n i}\right| \cdot\left|X_{1}\right| \geq \varepsilon\right\} \\
\leq & \sup _{n} \sum_{i=1}^{n} a_{n i}^{2} \cdot E X_{1}^{2} I\left\{\max _{1 \leq i \leq n}\left|a_{n i}\right| \cdot\left|X_{1}\right| \geq \varepsilon\right\} \rightarrow 0 \text { as } n \rightarrow \infty,
\end{aligned}
$$

thus the conclusion is proved by Lemma 2.4 .

Lemma 2.6 [2] Suppose that $f_{1}(x)$ and $f_{2}(y)$ are real, bounded, absolutely continuous functions on $R$ with $\left|f^{\prime}{ }_{1}(x)\right| \leq C_{1}$ and $\left|f^{\prime}{ }_{2}(y)\right| \leq C_{2}$. Then for any random variables $X$ and $Y$,

$$
\left|\operatorname{Cov}\left(f_{1}(X), f_{2}(Y)\right)\right| \leq C_{1} C_{2}\left\{-\operatorname{Cov}(X, Y)+8 p^{-}(X, Y)\|X\|_{2,1}\|Y\|_{2,1}\right\},
$$

where $\|X\|_{2,1}=\int_{0}^{\infty} P^{\frac{1}{2}}(|X|>x) d x$.

Lemma 2.7 Let $\left\{X_{n}, n \geq 1\right\}$ be a strictly stationary sequence of $\rho^{-}$-mixing random variables with $E X_{1}=0,0<E X_{1}^{2}<\infty$ and

$$
\begin{gathered}
0<\sigma_{1}^{2}=E X_{1}^{2}+2 \sum_{n=2}^{\infty} \operatorname{Cov}\left(X_{1}, X_{n}\right)<\infty, \\
\sum_{n=2}^{\infty}\left|\operatorname{Cov}\left(X_{1}, X_{n}\right)\right|<\infty,
\end{gathered}
$$


then for $0<p<2$, we have

$$
n^{-\frac{1}{p}} S_{n} \rightarrow 0 \text { a.s. as } n \rightarrow \infty \text {. }
$$

Proof By Lemma 2.1, we have

$$
\lim _{n \rightarrow \infty} \frac{E S_{n}^{2}}{n}=\sigma_{1}^{2} .
$$

Let $n_{k}=k^{\alpha}$, where $\alpha>\max \left\{1, \frac{p}{2-p}\right\}$. By (2.1), we get

$$
\sum_{k=1}^{\infty} P\left\{\left|S_{n k}\right| \geq \varepsilon n_{k}^{\frac{1}{p}}\right\} \leq \sum_{k=1}^{\infty} \frac{E S_{n_{k}}^{2}}{\varepsilon^{2} n_{k}^{\frac{2}{p}}} \leq \sum_{k=1}^{\infty} \frac{C}{\varepsilon^{2} k^{\alpha\left(\frac{2}{p}-1\right)}}<\infty .
$$

From Borel-Cantelli lemma, it follows that

$$
n_{k}^{-\frac{1}{p}} S_{n_{k}} \rightarrow 0 \text { a.s. as } k \rightarrow \infty .
$$

And by Lemma 2.2, it follows that

$$
\begin{aligned}
& \sum_{k=1}^{\infty} P\left\{\max _{n_{k} \leq n<n_{k+1}} \frac{\left|S_{n}-S_{n_{k}}\right|}{n^{\frac{1}{p}}} \geq \varepsilon\right\} \leq \sum_{k=1}^{\infty} \frac{E \max _{n_{k} \leq n<n_{k+1}}\left|S_{n}-S_{n_{k}}\right|^{2}}{\varepsilon^{2} n_{k}^{\frac{2}{p}}} \\
= & \sum_{k=1}^{\infty} \frac{E \max _{n_{k} \leq n<n_{k+1}}\left|\sum_{i=n_{k+1}}^{n} X_{i}\right|^{2}}{\varepsilon^{2} n_{k}^{\frac{2}{p}}} \leq C \sum_{k=1}^{\infty} \frac{\left(n_{k+1}-n_{k}\right)}{\varepsilon^{2} n_{k}^{\frac{2}{p}}} \leq C \sum_{k=1}^{\infty} \frac{1}{k^{\alpha}\left(\frac{2}{p}-1\right)}<\infty .
\end{aligned}
$$

By Borel-Cantelli lemma, we conclude that

$$
\max _{n_{k} \leq n<n_{k+1}} \frac{\left|S_{n}-S_{n_{k}}\right|}{n^{\frac{1}{p}}} \rightarrow 0 \text { a.s. as } n \rightarrow \infty .
$$

For every $n$, there exist $n_{k}$ and $n_{k+1}$ such that $n_{k} \leq n<n_{k+1}$, by (2.2) and (2.3), we have

$$
\begin{aligned}
\frac{\left|S_{n}\right|}{n^{\frac{1}{p}}} & =\frac{\left|S_{n}-S_{n_{k}}+S_{n_{k}}\right|}{n^{\frac{1}{p}}} \\
& \leq \frac{\left|S_{n_{k}}\right|}{n_{k}^{\frac{1}{p}}}+\max _{n_{k} \leq n<n_{k+1}} \frac{\left|S_{n}-S_{n_{k}}\right|}{n^{\frac{1}{p}}} \rightarrow 0 \text { a.s. as } n \rightarrow \infty .
\end{aligned}
$$

The proof is now completed.

\section{Proof of the theorems}

Proof of Theorem 1.1 By the property of $\rho^{-}$-mixing sequence, it is easy to see that $\left\{Y_{n}\right\}$ is a strictly stationary $\rho^{-}$-mixing sequence with $E Y_{1}=0$ and $E Y_{1}^{2}=1$. We first prove 


$$
\frac{S_{n, n}}{\sigma_{n}} \stackrel{d}{\rightarrow} \mathcal{N}(0,1) \text { as } n \rightarrow \infty .
$$

Let $a_{n i}=\frac{b_{i, n}}{\sigma_{n}}, 1 \leq i \leq n, n \geq 1$. Obviously,

$$
\operatorname{Var}\left(\sum_{i=1}^{n} a_{n i} Y_{i}\right)=1
$$

From condition $\left(a_{4}\right)$ in Theorem 1.1 and Lemma 2.3, we have

$$
\sup _{n} \sum_{i=1}^{n} a_{n i}^{2}=\sup _{n} \sum_{i=1}^{n} \frac{b_{i, n}^{2}}{\sigma_{n}^{2}}=\sup _{n} \frac{2 n-b_{1, n}}{\sigma_{n}^{2}} \leq C \sup _{n} \frac{2 n-b_{1, n}}{n}<\infty,
$$

and

$$
\max _{1 \leq i \leq n}\left|a_{n i}\right|=\max _{1 \leq i \leq n} \frac{b_{i, n}}{\sigma_{n}} \leq \frac{b_{1, n}}{\sigma_{n}} \leq \frac{C \log n}{\sqrt{n}} \rightarrow 0 \text { as } n \rightarrow \infty .
$$

By stationarity of $\left\{Y_{n}, n \geq 1\right\}$ and $\mathrm{E}\left|X_{1}\right|^{2}<\infty$, we know that $\left\{Y_{n}^{2}\right\}$ is uniformly integrable, and from condition $\left(a_{2}\right)$ in Theorem 1.1 , we get $\sum_{n=2}^{\infty}\left|\operatorname{Cov}\left(Y_{1}, Y_{n}\right)\right|<\infty$, so applying Lemma 2.5, we have

$$
\sum_{i=1}^{n} a_{n i} Y_{i} \stackrel{d}{\rightarrow} \mathcal{N}(0,1)
$$

Notice that

$$
\sum_{i=1}^{n} a_{n i} Y_{i}=\sum_{i=1}^{n} \frac{b_{i, n} Y_{i}}{\sigma_{n}}=\frac{S_{n, n}}{\sigma_{n}}
$$

so (3.1) is valid. Let $f(x)$ be a bounded Lipschitz function and have a Radon-Nikodyn derivative $h(x)$ bounded by $\Gamma$. From (3.1), we have

$$
E f\left(\frac{S_{n, n}}{\sigma_{n}}\right) \rightarrow E f(\mathcal{N}(0,1)) \text { as } n \rightarrow \infty,
$$

thus

$$
\frac{1}{\log n} \sum_{k=1}^{n} \frac{1}{k} E f\left(\frac{S_{k, k}}{\sigma_{k}}\right)-E f(\mathcal{N}(0,1)) \rightarrow 0 \text { as } n \rightarrow \infty .
$$

On the other hand, note that (1.1) is equivalent to

$$
\lim _{n \rightarrow \infty} \frac{1}{\log n} \sum_{k=1}^{n} \frac{1}{k} f\left(\frac{S_{k, k}}{\sigma_{k}}\right)=\int_{-\infty}^{\infty} f(x) d \Phi(x)=E f(\mathcal{N}(0,1)) \text { a.s. }
$$

from Section 2 of Peligrad and Shao [7] and Theorem 7.1 on $P_{42}$ from Billingsley [8]. Hence, to prove (3.3), it suffices to show that

$$
T_{n}=\frac{1}{\log n} \sum_{k=1}^{n} \frac{1}{k}\left[f\left(\frac{S_{k, k}}{\sigma_{k}}\right)-E f\left(\frac{S_{k, k}}{\sigma_{k}}\right)\right] \rightarrow 0 \text { a.s. } n \rightarrow \infty
$$


by (3.2). Let $\xi_{k}=f\left(\frac{S_{k, k}}{\sigma_{k}}\right)-E f\left(\frac{S_{k, k}}{\sigma_{k}}\right), 1 \leq k \leq n \mathrm{~m}$ we have

$$
\begin{aligned}
E T_{n}^{2} & =\frac{1}{\log ^{2} n} E\left(\sum_{k=1}^{n} \frac{\xi_{k}}{k}\right)^{2} \\
& \leq \frac{1}{\log ^{2} n} \sum_{1 \leq k \leq l \leq n, 2 k \geq l} \frac{\left|E \xi_{k} \xi_{l}\right|}{k l}+\frac{1}{\log ^{2} n} \sum_{1 \leq k \leq l \leq n, 2 k \geq l} \frac{\left|E \xi_{k} \xi_{l}\right|}{k l} \\
& :=I_{1}+I_{2} .
\end{aligned}
$$

By the fact that $\mathrm{f}$ is bounded, we have

$$
I_{1} \leq \frac{C}{\log ^{2} n} \sum_{k=1}^{n} \sum_{l=k}^{2 k} \frac{1}{k l}=\frac{C}{\log ^{2} n} \sum_{k=1}^{n} \frac{1}{k} \sum_{l=k}^{2 k} \frac{1}{l} \leq C\left(\log ^{-1} n\right) .
$$

Now we estimate $I_{2}$, if $l>2 k$, we have

$$
\begin{aligned}
S_{l, l}-S_{2 k, 2 k} & =\left(b_{1, l} Y_{1}+b_{2, l} Y_{2}+\cdots+b_{l, l} Y_{l}\right)-\left(b_{1,2 k} Y_{1}+b_{2,2 k} Y_{2}+\cdots+b_{2 k, 2 k} Y_{2 k}\right) \\
& =\left(b_{2 k+1, l} Y_{2 k+1}+\cdots+b_{l, l} Y_{l}\right)+b_{2 k+1,1} \tilde{S}_{2 k},
\end{aligned}
$$

and

$$
\begin{aligned}
\left|E \xi_{k} \xi_{l}\right| & =\left|\operatorname{Cov}\left(f\left(\frac{S_{k, k}}{\sigma_{k}}\right), f\left(\frac{S_{l, l}}{\sigma_{l}}\right)\right)\right| \\
& \leq\left|\operatorname{Cov}\left(f\left(\frac{S_{k, k}}{\sigma_{k}}\right), f\left(\frac{S_{l, l}}{\sigma_{l}}\right)-f\left(\frac{S_{l, l}-S_{2 k, 2 k}-b_{2 k+1, l} \tilde{S}_{2 k}}{\sigma_{l}}\right)\right)\right| \\
& +\left|\operatorname{Cov}\left(f\left(\frac{S_{k, k}}{\sigma_{k}}\right), f\left(\frac{S_{l, l}-S_{2 k, 2 k}-b_{2 k+1, l} \tilde{S}_{2 k}}{\sigma_{l}}\right)\right)\right| .
\end{aligned}
$$

By Lemma 2.3 and condition $\left(a_{2}\right)$ in Theorem 1.1, we have

$$
\begin{aligned}
\operatorname{Var}\left(S_{k, k}\right) & =\sum_{i=1}^{k} b_{i, k}^{2} E Y_{i}^{2}+2 \sum_{j=1}^{k-1} \sum_{i=j+1}^{k} b_{i, k} b_{j, k} \operatorname{Cov}\left(Y_{i}, Y_{j}\right) \\
& \leq \sum_{i=1}^{k} b_{i, k}^{2}+2 \sum_{j=1}^{k} b_{j, k}^{2} \sum_{i=j+1}^{k}\left|\operatorname{Cov}\left(Y_{i}, Y_{j}\right)\right| \\
& \leq C k,
\end{aligned}
$$

and

$$
\begin{aligned}
& \operatorname{Var}\left(S_{l, l}-S_{2 k, 2 k}-b_{2 k+1, l} \tilde{S}_{2 k}\right) \\
= & \sum_{i=2 k+1}^{l} b_{i, l}^{2} E Y_{i}^{2}+2 \sum_{j=2 k+1}^{l-1} \sum_{i=j+1}^{l} b_{i, l} b_{j, l} \operatorname{Cov}\left(Y_{i}, Y_{j}\right) \\
\leq & \sum_{i=2 k+1}^{l} b_{i, l}^{2}+2 \sum_{j=1}^{l} b_{i, l}^{2} \sum_{i=j+1}^{l}\left|\operatorname{Cov}\left(Y_{i}, Y_{j}\right)\right| \\
\leq & C l .
\end{aligned}
$$


By Lemma 2.6, the definition of $\rho^{-}$-mixing sequence and condition $\left(a_{4}\right)$, we have

$$
\begin{aligned}
&\left|\operatorname{Cov}\left(f\left(\frac{S_{k, k}}{\sigma_{k}}\right), f\left(\frac{S_{l, l}-S_{2 k, 2 k}-b_{2 k+1, l} \tilde{S}_{2 k}}{\sigma_{l}}\right)\right)\right| \\
& \leq C\left\{-\operatorname{Cov}\left(\frac{S_{k, k}}{\sigma_{k}}, \frac{S_{l, l}-S_{2 k, 2 k}-b_{2 k+1, l} \tilde{S}_{2 k}}{\sigma_{l}}\right)\right. \\
&\left.+8 \rho^{-}\left(\frac{S_{k, k}}{\sigma_{k}}, \frac{S_{l, l}-S_{2 k, 2 k}-b_{2 k+1, l} \tilde{S}_{2 k}}{\sigma_{l}}\right) \cdot\left\|\frac{S_{k, k}}{\sigma_{k}}\right\|_{2,1} \cdot\left\|\frac{S_{l, l}-S_{2 k, 2 k}-b_{2 k+1, l} \tilde{S}_{2 k}}{\sigma_{l}}\right\|_{2,1}\right\} \\
& \leq C \rho^{-}(k)\left(\operatorname{Var}\left(\frac{S_{k, k}}{\sigma_{k}}\right)\right)^{\frac{1}{2}}\left(\operatorname{Var}\left(\frac{S_{l, l}-S_{2 k, 2 k}-b_{2 k+1, l} \tilde{S}_{2 k}}{\sigma_{l}}\right)\right)^{\frac{1}{2}} \\
&+C \rho^{-}(k)\left\|\frac{S_{k, k}}{\sigma_{k}}\right\|_{2,1} \cdot\left\|\frac{S_{l, l}-S_{2 k, 2 k}-b_{2 k+1, l} \tilde{S}_{2 k}}{\sigma_{l}}\right\|_{2,1} \\
& \leq C \rho^{-}(k)+C \rho^{-}(k)\left\|\frac{S_{k, k}}{\sigma_{k}}\right\|_{2,1} \cdot\left\|\frac{S_{l, l}-S_{2 k, 2 k}-b_{2 k+1, l} \tilde{S}_{2 k}}{\sigma_{l}}\right\|_{2,1} \cdot
\end{aligned}
$$

By the inequality $\|X\|_{2,1} \leq \frac{r}{r-2}\|X\|_{r}(r>2)$ (cf. Zhang [[2], p. 254] or Ledoux and Talagrand [[9], p. 251]), we get

$$
\left\|\frac{S_{k, k}}{\sigma_{k}}\right\|_{2,1} \leq \frac{r}{r-2}\left\|\frac{S_{k, k}}{\sigma_{k}}\right\|_{r}=\frac{r}{r-2} \frac{1}{\sigma_{k}}\left(E\left|S_{k, k}\right|^{r}\right)^{\frac{1}{r}},
$$

and

$$
\begin{aligned}
E\left|S_{k, k}\right|^{r} & =E\left|\sum_{j=1}^{k} b_{j, k} Y_{j}\right|^{r} \\
& \leq C\left\{\sum_{j=1}^{k} b_{j, k}^{r} E\left|X_{j}\right|^{r}+\left(\sum_{j=1}^{k} b_{j, k}^{2} E X_{j}^{2}\right)^{\frac{r}{2}}\right\} \\
& \leq C\left\{k\left(\log ^{r} k\right)+k^{\frac{r}{2}}\right\},
\end{aligned}
$$

thus

$$
\left\|\frac{S_{k, k}}{\sigma_{k}}\right\|_{2,1} \leq C\left(\frac{r}{r-2} \cdot \frac{\log k}{k^{\frac{1}{2}-\frac{1}{r}}}+\frac{r}{r-2}\right)<C,
$$

similarly,

$$
\left\|\frac{S_{l, l}-S_{2 k, 2 k}-b_{2 k+1, l} \tilde{S}_{2 k}}{\sigma_{l}}\right\|_{2,1}<C,
$$

hence

$$
\left|\operatorname{Cov}\left(f\left(\frac{S_{k, k}}{\sigma_{k}}\right), f\left(\frac{S_{l, l}-S_{2 k, 2 k}-b_{2 k+1, l} \tilde{S}_{2 k}}{\sigma_{l}}\right)\right)\right| \leq C \rho^{-}(k) .
$$


Similarly to (3.7), we have

$$
\begin{aligned}
\operatorname{Var}\left(S_{2 k, 2 k}\right) & =\sum_{i=1}^{2 k} b_{i, 2 k}^{2} E Y_{i}^{2}+2 \sum_{j=1}^{2 k-1} \sum_{i=j+1}^{2 k} b_{i, 2 k} b_{j, 2 k} \operatorname{Cov}\left(Y_{i}, Y_{j}\right) \\
& \leq \sum_{i=1}^{2 k} b_{i, 2 k}^{2}+2 \sum_{j=1}^{2 k-1} b_{i, 2 k}^{2} \sum_{i=j+1}^{2 k}\left|\operatorname{Cov}\left(Y_{i}, Y_{j}\right)\right| \\
& \leq C k,
\end{aligned}
$$

and

$$
\begin{aligned}
\operatorname{Var}\left(\tilde{S}_{2 k}\right) & =\operatorname{Var}\left(\sum_{i=1}^{2 k} Y_{i}\right) \\
& =\sum_{i=1}^{2 k} E Y_{i}^{2}+2 \sum_{i=1}^{2 k-1} \sum_{j=i+1}^{2 k}\left|\operatorname{Cov}\left(Y_{i}, Y_{j}\right)\right| \\
& =2 k+2 \sum_{i=1}^{2 k-1} \sum_{j=2}^{2 k-i+1} \operatorname{Cov}\left(Y_{i}, Y_{j}\right) \\
& \leq C k .
\end{aligned}
$$

Since $f$ is a bounded Lipschitz function, we have

$$
\begin{aligned}
& \left|\operatorname{Cov}\left(f\left(\frac{S_{k, k}}{\sigma_{k}}\right), f\left(\frac{S_{l, l}}{\sigma_{l}}\right)-f\left(\frac{S_{l, l}-S_{2 k, 2 k}-b_{2 k+1, l} \tilde{S}_{2 k}}{\sigma_{l}}\right)\right)\right| \\
\leq & C E \frac{\left|S_{2 k, 2 k}+b_{2 k+1, l} \tilde{S}_{2 k}\right|}{\sigma_{l}} \\
\leq & C \frac{\left(\operatorname{Var}\left(S_{2 k, 2 k}\right)\right)^{\frac{1}{2}}}{\sigma_{l}}+C \frac{b_{2 k+1, l}\left(\operatorname{Var}\left(\tilde{S}_{2 k}\right)\right)^{\frac{1}{2}}}{\sigma_{l}} \\
\leq & C\left(\frac{k}{l}\right)^{\frac{1}{2}}+C\left(\frac{k}{l}\right)^{\frac{1}{2}} \log \frac{1}{2 k} \\
\leq & C\left(\frac{k}{l}\right)^{\varepsilon},
\end{aligned}
$$

where $0<\varepsilon<\frac{1}{2}$. Hence if $l>2 k$, we have

$$
\left|E \xi_{k} \xi_{l}\right| \leq C\left[\rho^{-}(k)+\left(\frac{k}{l}\right)^{\varepsilon}\right] \text {. }
$$


Thus

$$
\begin{aligned}
I_{2} & \leq \frac{C}{\log ^{2} n} \sum_{l=2}^{n} \sum_{k=1}^{l-1} \frac{1}{k^{1-\varepsilon} l^{1+\varepsilon}}+\frac{C}{\log ^{2} n} \sum_{l=2}^{n} \frac{1}{l} \sum_{k=1}^{l-1} \frac{\rho^{-}(k)}{k} \\
& \leq \frac{C}{\log ^{2} n} \sum_{l=2}^{n} \frac{1}{l^{1+\varepsilon}} \frac{(l-1)^{\varepsilon}}{\varepsilon}+\frac{C}{\log ^{2} n} \sum_{l=2}^{n} \frac{1}{l} \sum_{k=1}^{n} \frac{\log ^{-\delta} k}{k} \\
& \leq \frac{C}{\log ^{2} n} \sum_{l=2}^{n} \frac{1}{l}+\frac{C}{\log ^{2} n} \sum_{l=2}^{n} \frac{1}{l} \sum_{k=1}^{n} \frac{\log ^{-\delta} k}{k} \\
& \leq \log ^{-1} n .
\end{aligned}
$$

Associated with (3.5), (3.6), and (3.8), we have

$$
E T_{n}^{2} \leq C \log ^{-1} n .
$$

To prove (3.4), let $n_{k}=e^{k^{\tau}}$, where $\tau>1$. From (3.9), we have

$$
\sum_{k=1}^{\infty} E T_{n_{k}}^{2} \leq C \sum_{k=1}^{\infty} \log ^{-1} n_{k}=C \sum_{k=1}^{\infty} \frac{1}{k^{\tau}}<\infty .
$$

Thus $\forall \varepsilon>0$, we have

$$
\sum_{k=1}^{\infty} P\left\{\left|T_{n_{k}}\right| \geq \varepsilon\right\} \leq \sum_{k=1}^{\infty} \frac{E T_{n_{k}}^{2}}{\varepsilon^{2}}<\infty .
$$

By Borel-Cantelli lemma, we have

$$
T_{n_{k} \rightarrow 0} \text { a.s. as } k \rightarrow \infty .
$$

Note that

$$
\frac{\log n_{k+1}}{\log n_{k}}=\frac{(k+1)^{\tau}}{k^{\tau}} \rightarrow 1 \text { as } k \rightarrow \infty .
$$

For every $n$, there exist $n_{k}$ and $n_{k+1}$ satisfying $n_{k}<n \leq n_{k+1}$, we have

$$
\begin{aligned}
\left|T_{n}\right| & \leq \frac{1}{\log n_{k}}\left|\sum_{i=1}^{n_{k}} \frac{\xi_{i}}{i}\right|+\frac{1}{\log n_{k}} \sum_{i=n_{k}}^{n_{k+1}} \frac{\left|\xi_{i}\right|}{i} \\
& \leq\left|T_{n_{k}}\right|+C\left(\frac{\log n_{k+1}}{\log n_{k}}-1\right) \rightarrow 0 \text { a.s. as } n \rightarrow \infty,
\end{aligned}
$$

(3.4) is completed, so the proof of Theorem 1.1 is completed.

Proof of Theorem 1.2 Let $C_{i}=\frac{S_{i}}{\mu_{i}}$, we have

$$
\frac{1}{\gamma \sigma_{k}} \sum_{i=1}^{k}\left(C_{i}-1\right)=\frac{1}{\gamma \sigma_{k}} \sum_{i=1}^{k}\left(\frac{S_{i}}{\mu i}-1\right)=\frac{1}{\sigma_{k}} \sum_{i=1}^{k} b_{i, k} Y_{i}=\frac{S_{k, k}}{\sigma_{k}} .
$$

Hence (1.1) is equivalent to

$$
\forall x \lim _{n \rightarrow \infty} \frac{1}{\log n} \sum_{k=1}^{n} \frac{1}{k} I\left\{\frac{1}{\gamma \sigma_{k}} \sum_{i=1}^{k}\left(C_{i}-1\right) \leq x\right\}=\Phi(x) \text { a.s. }
$$


On the other hand, to prove (1.2), it suffices to show that

$$
\forall x \quad \lim _{n \rightarrow \infty} \frac{1}{\log n} \sum_{k=1}^{n} \frac{1}{k} I\left\{\frac{1}{\gamma \sigma_{k}} \sum_{i=1}^{k} \log C_{i} \leq x\right\}=\Phi(x) \text { a.s. }
$$

By Lemma 2.7, for enough large $i$, for some $\frac{4}{3}<p<2$ we have

$$
\left|C_{i}-1\right|=\left|\frac{S_{i}}{\mu i}-1\right| \leq C i^{\frac{1}{p}-1} \text { a.s. }
$$

It is easy to know that $\log (1+x)=x+O\left(x^{2}\right)$ for $|x|<\frac{1}{2}$, thus

$$
\left|\sum_{k=1}^{n} \log C_{k}-\sum_{k=1}^{n}\left(C_{k}-1\right)\right| \leq C \sum_{k=1}^{n}\left(C_{k}-1\right)^{2} \leq C \sum_{k=1}^{n} k^{\frac{2}{p}-2} \leq C n^{\frac{2}{p}-1} \text { a.s., }
$$

and

$$
\sum_{k=1}^{n}\left(C_{k}-1\right)-C n^{\frac{2}{p}-1} \leq \sum_{k=1}^{n} \log C_{k} \leq \sum_{k=1}^{n}\left(C_{k}-1\right)+C n^{\frac{2}{p}-1} \text { a.s. }
$$

Hence for arbitrary small $\varepsilon>0$, there is $n_{0}=n_{0}(\omega, \varepsilon)$, such that for every $n>n_{0}$ and arbitrary $x$,

$$
I\left\{\frac{1}{\gamma \sigma_{k}} \sum_{i=1}^{k}\left(C_{i}-1\right) \leq x-\varepsilon\right\} \leq I\left\{\frac{1}{\gamma \sigma_{k}} \sum_{i=1}^{k} \log C_{i} \leq x\right\} \leq I\left\{\frac{1}{\gamma \sigma_{k}} \sum_{i=1}^{k}\left(C_{i}-1\right) \leq x+\varepsilon\right\},
$$

so by (3.10), we know that (3.11) is true, and (3.11) is equivalent to (1.2), thus the proof of Theorem 1.2 is complete.

\section{Acknowledgements}

The authors were very grateful to the editor and anonymous referees for their careful reading of the manuscript and valuable suggestions which helped in significantly improving an earlier version of this article. The study was supported by the National Natural Science Foundation of China (10926169, 11171003, 11101180), Key Project of Chinese Ministry of Education (211039), Foundation of Jilin Educational Committee of China (2012-158), and Basic Research Foundation of Jilin University (201001002, 201103204).

\section{Author details}

${ }^{1}$ Department of Mathematics, Beihua University, Jilin 132013, P.R. China ${ }^{2}$ College of Mathematics, Jilin University, Changchun 130012, P.R. China

\section{Authors' contributions}

$X T$ and $Y Z$ carried out the design of the study and performed the analysis. YZ participated in its design and coordination. All authors read and approved the final manuscript.

\section{Competing interests}

The authors declare that they have no competing interests.

Received: 13 November 2011 Accepted: 1 March 2012 Published: 1 March 2012

\section{References}

1. Zhang, LX, Wang, XY: Convergence rates in the strong laws of asymptotically negatively associated random fields. Appl Math J Chinese Univ Ser B. 14(4):406-416 (1999). doi:10.1007/s11766-999-0070-6

2. Zhang, LX: A functional central limit theorem for asymptotically negatively dependent random fields. Acta Math Hungar. 86(3):237-259 (2000). doi:10.1023/A:1006720512467

3. Zhang, LX: Central limit theorems for asymptotically negatively associated random fields. Acta Math Sinica. 6(4):691-710 (2000)

4. Wang, JF, Lu, FB: Inequalities of maximum of partial sums and weak convergence for a class of weak dependent random variables. Acta Math Sinica. 22(3):693-700 (2006). doi:10.1007/s10114-005-0601-x 
5. Zhou, H: Note on the almost sure central limit theorem for $\rho$-mixing sequences. J Zhejiang Univ Sci Ed. 32(5):503-505 (2005)

6. Khurelbaatar, G, Rempala, G: A note on the almost sure central limit theorem for the product of partial sums. Appl Math Lett. 19(2):191-196 (2006). doi:10.1016/j.aml.2005.06.002

7. Peilgrad, M, Shao, QM: A note on the almost sure central limit theorem. Statist Probab Lett. 22, 131-136 (1995). doi:10.1016/0167-7152(94)00059-H

8. Billingsley, P: Convergence of Probability Measures. Wiley, New York (1968)

9. Ledoux, M, Talagrand, M: Probability in Banach Space. Springer Verlag, New York (1991)

doi:10.1186/1029-242X-2012-51

Cite this article as: Tan et al: An almost sure central limit theorem of products of partial sums for $\boldsymbol{\rho}$-mixing sequences. Journal of Inequalities and Applications 2012 2012:51.

Submit your manuscript to a SpringerOpen ${ }^{\circ}$ journal and benefit from:

- Convenient online submission

- Rigorous peer review

- Immediate publication on acceptance

- Open access: articles freely available online

- High visibility within the field

- Retaining the copyright to your article

Submit your next manuscript at $\boldsymbol{\triangleright}$ springeropen.com 